\title{
Timer based Location Management in Cellular Network using Cache Memory
}

\author{
Ramratan Ahirwal \\ Computer Science \& Engineering Dept. \\ S.A.T.I., Vidisha, India
}

\author{
Alok Sahelay \\ Computer Science \& Engineering \\ S.A.T.I., Vidisha, India
}

\begin{abstract}
Location management is essential task in current cellular system. Mobility prediction is widely used to assist handoff management, resource reservation and service preconfiguration. Location management methods are to find out mobile unit current location. Location update and paging have to maintain efficiently to minimize location management cost in cellular network. This paper introduce new timer based algorithm using cache memory for reducing paging cost. This algorithm is based on user's daily predefined moving geographical activities pattern, according to time. Paging decision for user is based on this predicted location for any instance of time interval. This predicated value again sort by higher probability of user finding in any cell for that time duration. This prediction information is saved by mobile unit in its personal cache memory for every fixed time interval. If more prediction value searched for paging, so it increases average paging cost. Therefore here is threshold value used for maintain page hit ratio. But if threshold value is to less hit ratio comes down rapidly for smaller threshold values. Thus threshold value helps to maintain hit page, hit to page miss ratio. The results confirm the effectiveness of this method compare to existing method for real time in mobile services and proposed method is $6 \%$ more efficient in average hit ratio.
\end{abstract}

\section{Keywords}

Location Management, Location Update, Paging, Cellular network, Time-slot, Mobile switching center (MSC).

\section{INTRODUCTION}

Recent advances in cellular mobile communications have touched almost every aspect of our lives. This wide cellular communication has need to expanded of a new generation mobile communication network called personal communications services (PCS), which help us to support larger mobile user population while servicing various types of services, which was unavailable to conventional cellular systems. PCS networks offer wireless communication services that enable mobile terminals (MTs) to transfer any information to any location at any instance. To provide the services timely, keeping track the location of mobile users is necessary. It is a challenging task to perform this mechanism efficiently and timely. Usually this mechanism is referred to as Location management, which is one of the key issues in today's cellular network. This paper introduce new location management scheme based on user daily routine activity according to time.

Location management refers as how to track the MTs that move from one place to other place in PCS networks [14], [15], [16]. Two-tier system is used in PCS networks for the location management, one of Home Location Register (HLR) databases and Visitor Location Register (VLR) databases. The HLR contains the permanent data (e.g., directory number, profile information, current location, and validation period) of the MTs whose primary subscription is within the area. For each MT, it contains a pointer to the VLR to assist routing incoming calls. A VLR is associated with a Mobile Switching Center (MSC) in the networks as shown in fig.1. It contains temporary record for all MTs currently active within the service area of the MSC. The VLR retrieves information for handling calls to MT or from a visiting MT. To facilitate the tracking of a moving MT, a PCS network is partitioned into many registration area (RA) or location areas (LAs). Each LA may include tens or hundreds of cells, which is a basic unit of area served by a base station (BS). Each LA is serviced by a VLR. An HLR is associated with tens or hundreds of VLRs. The service area served by an HLR is referred to as Service Area (SA).

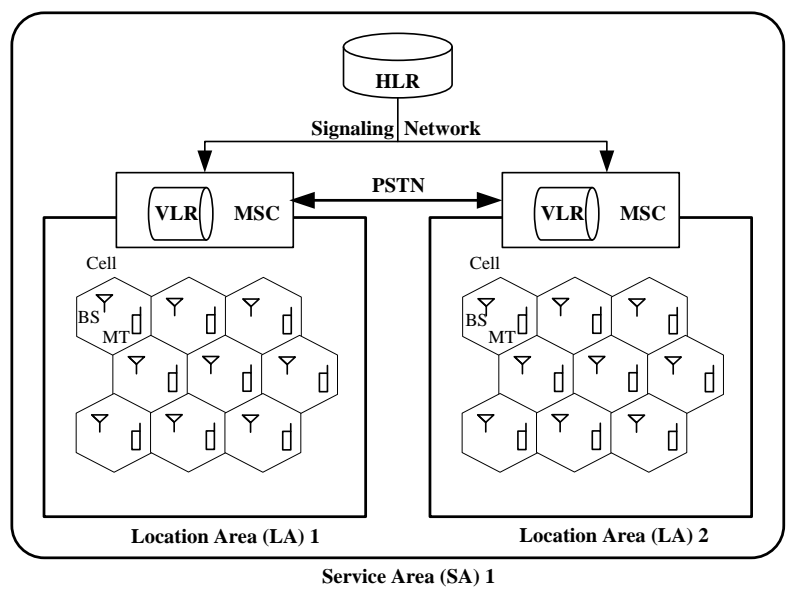

Fig 1: Cellular network architecture

Location management handles current position of any mobile user it tracks the mobile terminal during servicing call arrivals and makes sure that the call is shifted without disconnecting the call. Cellular network is based on three major components Mobile unit (MT), Base station (BS) and Location area (LA). MT is an electronic device used for sending and receiving data signals. Service area is divided in to LA and every LA is divided in to BS which has a station for receiving and sending signals for MT. Mobile unit uses pre-assigned radio frequencies [1], [2] set for communicating voice or data signals. Location of MT can be retrieve by Registration or Location Update and Paging or Polling.

The two basic operations involved in location management mechanism are Location update and Paging [7]. Location update is concerned with the reporting of current cell location by the mobile user. When an incoming call arrives for a mobile user, the network searches the user by sending polling signals in a cell or a number of cells, where the mobile user is most likely to be found [6]; this searching process is known as paging. In static location management, cells are constant in 
shape and size, and identical for every mobile user. Every mobile user moving through them follows same set of location management rules. Three basic static location update schemes are Always-update, Never-update and static intervalbased update. Practically, the third of these schemes is most commonly used [5]. It is a good method for location updating. Cells may differ in shape and size, and may not be identical for every mobile user. Three basic dynamic location update schemes are Time-based, Movement-based and Distancebased update [8]. To get access of current location as soon as possible, various methods of paging have been proposed in which most basic method used is simultaneous paging. In this method every cell for user's location area is paged at the same time in order to find current location. An another scheme is Sequential paging, where each cell within a location area is paged, with one common theory suggesting the polling of small cell areas in order of decreasing user dwelling probability [3]. The total location management cost $\left(C_{\text {Total }} L M\right)$ can be calculated by adding location update operation cost $\left(C_{\text {Update }}\right)$ and paging operation cost $\left(C_{\text {Paging }}\right)$.

$$
C_{\text {Total }} L M=C \times C_{\text {Update }}+C_{\text {Paging }}
$$

Here $C_{\text {Total }} L M$ is the total location management cost [10]. $C_{\text {Update }}$ is the number of LU messages generated $C_{\text {Paging }}$ is the number of cells paged. $\mathrm{C}$ is constant representing the relative cost of update to pages and is taken as 1 , roughly representing the approximate size and number of signaling messages required by LU as compared to paging message.

Generally speaking, Location update (LU) costs are higher than paging costs. The network can require more frequent LUs, in order to reduce paging costs. Conversely, the network could only require rare LUs, storing less information about users to reduce computational overhead, but at a higher polling cost. To reduce the total location management cost, it is necessary to provide a good tradeoff between the paging and location update operations [6].

\section{PREVIOUS WORK}

The LU procedures can be classified into static and dynamic schemes. In the static schemes, the whole coverage area is divided into several fixed RAs called LAs. Each LA is a fixed group of neighboring cells, so that the MT delivers an LU message to the network whenever it crosses an LA border. In dynamic strategies, the RA borders depend on the cell where the MT position was updated for the last time. Current Cellular networks are partitioned into number of LAs. The LU is performed when a current terminal enters a new LA. When a call arrives, the network locates the MS by simultaneously paging all the cells of the current LA of the mobile terminal. The static interval-based update algorithm [5] attempts utilize the mobility history of the subscriber to dynamically create LA for individual subscribers. This algorithm dynamically determines the most probable paging areas by maintaining the time duration for which a subscriber stayed in the cell, for all the cells in the current LA.

Proper Resource utilization can be done by efficient paging of LA because MT moves frequently form current location so it is costly to page whole LA every time., many LM schemes use previous record of MT to predict a set of probable current locations of goal MT. MT is located by predefined set of cell ID based on time according user's moving pattern, it minimize paging cost, by using predefined set of cell ID based scheme reduce great average location management cost. To reduce the total location management cost, it is necessary to provide a good tradeoff between the paging and location update operations [6]. In another method MT is searched simultaneously in the set of predicted cells wherever if it is found then called a page hit otherwise it is a page miss and in this case, MT is searched at the same time in all the left over cells of its present LA. If system can predict user's location exactly then the paging cost is reduced a lot [9] this method is very efficient to minimize total cost of location management. Another method gives better LM scheme by dynamic location management scheme using user speed based algorithm [10].

Another latest research done objective of this research is to propose, validate and evaluate the performance of location update scheme using fuzzy logic technique [21] for cellular radio system. Three metrics: speed, user density and residence time are proposed and used for fuzzy evaluation. The results of using fuzzy logic technique show that location management cost reduction using fuzzy logic in cellular radio network using fuzzy logic is decreased about $2 \%$ and outperforms the conventional mechanism. New work done based on geometric location proposed in Geometrical positioning approached for mobile location estimation method [20] This paper illustrates hybrid proposed schemes that combine time of arrival (TOA) at three BSs and angle of arrival (AOA) information at the serving BS to give a location estimate of the MS. The proposed schemes mitigate the NLOS effect simply by the weighted sum of the intersections between three TOA circles and the AOA line without requiring a priori information about the NLOS error. Simulation results show that the proposed methods can achieve better accuracy when compare with Taylor series algorithm (TSA) and the hybrid lines of position algorithm (HLOP). Study of Timer Based Method for Location Management with the Blocking Probability is very helpful for new work in this field [19]. A good time based method is given in paper [18] proposes an activity based mobility prediction technique that uses activity prediction and Markov modeling techniques (AMPuMM) to devise a prediction methodology that could make accurate predictions than existing techniques.

The research in the area of mobile location management is Speed based adaptive method [10]. This technique keeps track record of user movement with its moving speed and LA is defined according to mobile user's speed. Time based dynamic algorithm [3] is find MT location according to its time period prediction which gives very optimum result for working days.

\section{TIME BASED LOCATION MANAGEMENT}

Through various surveys it had been observed that most of user activities occur only at particular locations, for fixed time periods. The geographical activities of individual are found to be steady over longer periods of time while the time-sequence of stay and repeatability has a shorter life span. Based on these facts this paper proposes a time based location prediction scheme for that time slot could reflect changes in user's behavior earlier than other pattern based techniques and could provide accurate predictions on MT's future locations [17].

This section proposed a new LM scheme in which current location of MT is predict by previous data stored at VLR in form of table called mobility data table (MDT). MDT has maintained particular user's location within every time slot. Here we are considering time slot of one hour, so MT have cache memory in mobile unit to keep record cell ID for every hour. At starting of every time slot MT store its current cell ID in corresponding time slot in form of MDT at MT side in 
its cache memory. This MDT send to VLR database to keep all day location stay for this MT. VLR side MDT gets updated by sending of MDT through MT at mid night when channel become ideal and control signal can be transmitted for better resource utilization. Due to mid night MDT transmission, frequent registration is not required, hence registration cost become near about constant (very low). If this data transmitted in day time then it can consume resource which is used for data transmission and average LM cost could increase, MT store cell ID at every time slot interval and store in its cache memory, at mid night this whole information is transferred to VLR for updating VLR data in VLR side MDT as table-1. MDT maintains user information according the time, time is divided into slot for every hour and VLR keeps record of every cell ID according time slot. This table can be given as follow:

Table 1. Mobile Data Table (MDT)

\begin{tabular}{|c|c|c|}
\hline Slot No. & Hours & Prediction Sets \\
\hline 0 & $00: 00$ to $01: 00$ & {[]} \\
\hline 1 & $01: 00$ to $02: 00$ & {[]} \\
\hline .. & $\ldots$ & $\ldots$ \\
\hline .. & $\ldots$ & $\ldots$ \\
\hline 23 & $23: 00$ to $24: 00$ & {[]} \\
\hline
\end{tabular}

Initially prediction sets having only one cell ID which is MT's home cell ID for each time slot. When call is arrived MT, VLR select sets by corresponding time slot at which time call is arrived and then get index 1 cell ID of that set because this slot maintain most prior cell at index 1 by applying automatic sorting on set. If MT not found in index 1 ID of predicted set than traditional paging applied and current cell ID is inserted into prediction set if user again found in this ID again at same time slot than this ID is swapped with nearest left cell ID. Now next time this shifted ID becomes at index 1 and paged first on call if arrived in this time slot. This table is maintained by MT with help of cache memory at MT.

The second column of MDT holds an entry for each hour of the day whereas subsequent column indicates the set of predicted cells where the MT can probably be present. Initially, the network has no mobility information of MT therefore second column is fully empty and in this situation. Overall location management cost can be calculated by registration cost and paging cost. Above registration method can explain easily by following timer based registration algorithm. When call arrives and VLR searches for Mobile terminal then paging process will call and search for MT (let us say between time $[\mathrm{T}]$ to $[\mathrm{T}+1])$ then whole LA will be paged simultaneously and the cell-id of the cell where MT is found (lets us say C3) will be inserted in the corresponding time in MDT as follow:

$$
[T] \text { to }[T+1] \rightarrow[C 3]
$$

Next time when a call arrives for this MT between duration [T] to [T+1] then firstly MT will be first searched in cell C3 and if MT is found (page hit occurs) then no update will take place in MDT prediction set for this duration ([T] to $[\mathrm{T}+1])$. MDT is updated only when a page miss occurs. Let us assume that MT was present in cell C5 when next call arrived and a page miss occurred. Now MDT will add C5 as the last (rightmost) entry in its prediction set.

$$
[T] \text { to }[T+1] \rightarrow[C 3, C 5]
$$

Following the above scheme may lead to a large prediction set, which eventually will lead to high paging cost. Therefore the maximum number of cells that can be paged in the first phase is determined by threshold $\mathrm{N}$, which means at most only the first $\mathrm{N}$ cells of prediction set can be paged in the first phase of paging. For example at any time, if $\mathrm{N}=5$ (as shown by underlined elements) and MDT contains following entry:

$$
[T] \text { to }[T+1] \rightarrow[C 3, C 5, C 1, C 9, C 23, C 18, C 15, C 21]
$$

A call arrives for MT between this duration, then firstly it will be searched only in cell $\mathrm{C} 3, \mathrm{C} 5, \mathrm{C} 1, \mathrm{C} 9$ and $\mathrm{C} 23$ i.e. the first five (left to right) cells in the prediction set. If a page hit occurs then no changes are made in MDT but if a page miss occurs then all the remaining cells in the LA will be searched simultaneously to locate MT and MDT will be reorganized according to following procedure:

1) If MT is found in a cell which is not present in its prediction set then:

a) If size of prediction set is greater than $\mathrm{N}$ then new cell acquires last (rightmost) position in prediction set.

b) If size of prediction set is greater than or equal to $\mathrm{N}$ then new cell acquires $\mathrm{N}^{\text {th }}$ position in prediction set.

2) If MT is found in a cell which is already present in its prediction set then a left shift takes place in the position of that cell.

For example, if a page miss occurs and MT is found in cell $\mathrm{C} 12$, which is not present in MDT then it will be inserted immediately after $\mathrm{C} 9$ i.e. at $5^{\text {th }}$ position (since $\mathrm{N}$ is taken as 5).

$$
[T] \text { to }[T+1] \rightarrow[C 3, C 5, C 1, C 9, C 12, C 23, C 18, C 15, C 21]
$$

Whereas, if a page miss occurs and MT is found in cell C23, then the positions of $\mathrm{C} 23$ and $\mathrm{C} 12$ will be interchanged.

$$
[T] \text { to }[T+1] \rightarrow[C 3, C 5, C 1, C 9, C 23, C 12, C 18, C 15, C 21]
$$

Now cell $\mathrm{C} 23$ is one of the first five cells and therefore next time when a call arrives in duration $[\mathrm{T}]$ to $[\mathrm{T}+1]$, cell $\mathrm{C} 23$ will also be paged. This paging method can be explained by following algorithm.

\section{PROPOSED ALGORITHM}

\subsection{Algorithm for Timer-Based using cache memory Registration Scheme}

1. When a mobile terminal is turned on,

a) it updates its current location to VLR

b) Store current location id in its cache memory for current time slot at first index.

c) Sets the cell movement cell_counter=1 for this time slot.

d) Store default cell ID for every time slot in mobile cache MDT.

2. When a mobile user enters new cell

a) MT checks current cell ID in cache MDT for current time slot-

If the ID is not found in MT's MDT then

i) MT inserts this new cell ID for this time slot.

ii) Increment the value of cell_counter by 1 .

Else if the ID is found in its cache MDT then

i) Checks position of this ID in cache array. 
ii) Swap its position by nearest left cell ID.

3. When the value of cell_counter reaches the movement threshold D or time is 00:00(mid-night)

a) MT updates its current location.

b) Copies the contents of the cache to the VLR.

4. When a mobile terminal enters to new VLR then

a) New VLR sends the de_registration message to the old VLR.

b) Old VLR forwards the mobility information of the mobile terminal to the new VLR.

c) Old VLR deletes all records of this MT after receiving acknowledgment

d) New VLR updates the mobility information of this mobile user.

\subsection{Algorithm for Timer-Based using cache memory Paging Scheme}

1. When an incoming call arrives

a) The system retrieves the record which has the mobility information MDT for the target MT in the VLR database

b) Performs the timer-based paging to current time slot cells in the list of the MDT.

2. Once record fetched then

a) Target MT is searched in first entry of MDT at VLR for current time slot.

b) If not found in first entered cell ID then Check for all next entry in this time slot.

3. If the target mobile terminal is not found in timer-based paging process then normal traditional location area paging is performed again to find the target mobile terminal.

The proposed LM scheme inserts a new cell-id at Nth position because from here, this cell can be removed from first paging phase and this position can be occupied by a more frequently visited cell in only one shift operation. The left shift in already existing cell makes its position nearer to $\mathrm{N}$ each time a call arrives, thus the most frequently visited cells are always paged in first phase of paging without increasing the number of predicted cells.

\section{Simulation and Results}

\subsection{Network Model}

For simulation purpose, a $15 \times 15$ network is modeled as shown in Fig.2. Each cell is assigned a unique number and is used as its cell-id. There are 225 cells in the network, which are partitioned into 9 LAs shown with different levels of darkness. Each LA consists of 25 cells as given below:

- First LA consists of Cell 0, 1, 2, 3, 4, 15, 16, 17, 18, 19, $30,31,32,33,34,45,46,47,48,49,60,61,62,63$, and 64.

- Second LA consists of Cell 5, 6, 7, 8, 9, 20, 21, 22, 23, $24,35,36,37,38,39,50,51,52,53,54,65,66,67,68$, and 69.

- Such as Ninth LA consists of Cell 160, 161, 162, 163, $164,175,176,177,178,179,190,191,192,193,194$, 205, 206, 207, 208, 209, 220, 221, 222, 223, and 224.

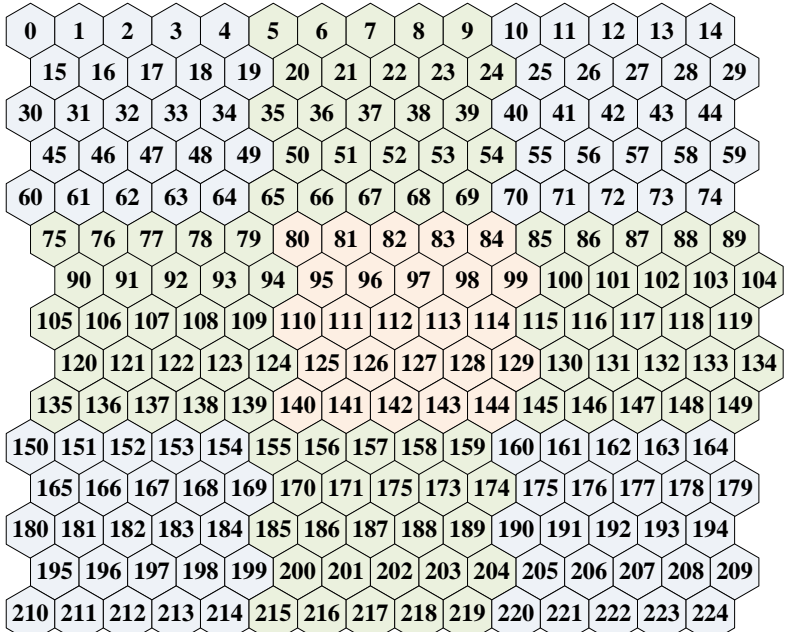

Fig 2: A 15×15 network with 9 LAs

Initially all the prediction sets are empty, which are gradually filled depending upon the call arrival frequencies and mobility patterns of each MT. We have simulated the network with 500 MTs for duration of eleven days. An MT triggers a location update on changing the LA. For evaluation purpose, we have examined proposed scheme on various parameters at different threshold values and without threshold. For mobility model, we assume the daily worker mobility pattern [6] for time 08:00am to $05: 00 \mathrm{pm}$ after which, user can move at semirandom. MT travels almost the same route everyday between home, gymnasium and work locations whereas from 05:00pm to $11: 00 \mathrm{pm}$, the user may move randomly. This is done in order to model actual user movement because most of the users follow daily routine pathway to gymnasium, home, workplace, college, school etc. and after which, they may move freely.

\subsection{Call Arrival Pattern}

It has been observed that among all calls generated to a subscriber during a whole day [3], 20 percent calls are generated in between 00:00 to 08:00, 50 percent calls are generated in between 08:00 to 18:00 and remaining 30 percent calls are generated in between 18:00 to 24:00.Based on this, the call arrival probability is shown in Table 2.

Table 2. Call arrival Probabilities

\begin{tabular}{|c|c|}
\hline Time of day & Probability \\
\hline 00:00 to $08: 00$ & 0.2 \\
\hline 08:00 to $18: 00$ & 0.50 \\
\hline 18:00 to $24: 00$ & 0.30 \\
\hline
\end{tabular}

\subsection{Paging Cost}

Increasing threshold value means to increase the number of cells where an MT will be searched during the first phase of paging; although it increases possibility of finding MT in first phase but it also raises total paging cost as each time a call arrives, the minimum number of cells that needs to be paged increases.

$C_{\text {Paging }}=S \times\left\{P_{\text {hit }} \times N_{\text {cache }}+\left(1-P_{\text {hit }}\right) \times N_{\text {area }}\right\} \times C_{\text {page }}$

Where, $P_{\text {hit }}=$ Probability that the target mobile terminal exists in the cell which is included in the mobility information for the mobile user in the VLR.

$S=$ Total number of calls attempted per hour in the system. 
$N_{\text {cache }}=$ Number of cells to be paged in the selective paging.

$N_{\text {area }}=$ Number of cells to be paged in the location area paging.

$C_{\text {page }}=$ Unit paging cost

For simulation purpose, the unit paging cost is taken as 1 . The proposed scheme is simulated with five different thresholds and without any threshold value.

Table 3. Paging Cost for different Threshold value

\begin{tabular}{|c|c|c|c|c|c|}
\hline Days & Without $\mathbf{T h}$ & $\mathbf{T h}=\mathbf{8}$ & $\mathbf{T h}=\mathbf{6}$ & $\mathbf{T h}=\mathbf{4}$ & $\mathbf{T h = 2}$ \\
\hline 1 & 77788 & 64423 & 57719 & 54403 & 54962 \\
\hline 2 & 27610 & 23021 & 22628 & 22294 & 24035 \\
\hline 3 & 28226 & 23182 & 22093 & 22523 & 23595 \\
\hline 4 & 28667 & 23386 & 21431 & 22627 & 24575 \\
\hline 5 & 32828 & 26611 & 22945 & 23104 & 23620 \\
\hline 6 & 34271 & 27747 & 22869 & 21923 & 14199 \\
\hline 7 & 37914 & 30640 & 24200 & 19412 & 11412 \\
\hline 8 & 66876 & 53814 & 41374 & 30555 & 20194 \\
\hline 9 & 42966 & 34604 & 26712 & 19580 & 11694 \\
\hline 10 & 68162 & 54831 & 42025 & 29635 & 12236 \\
\hline
\end{tabular}

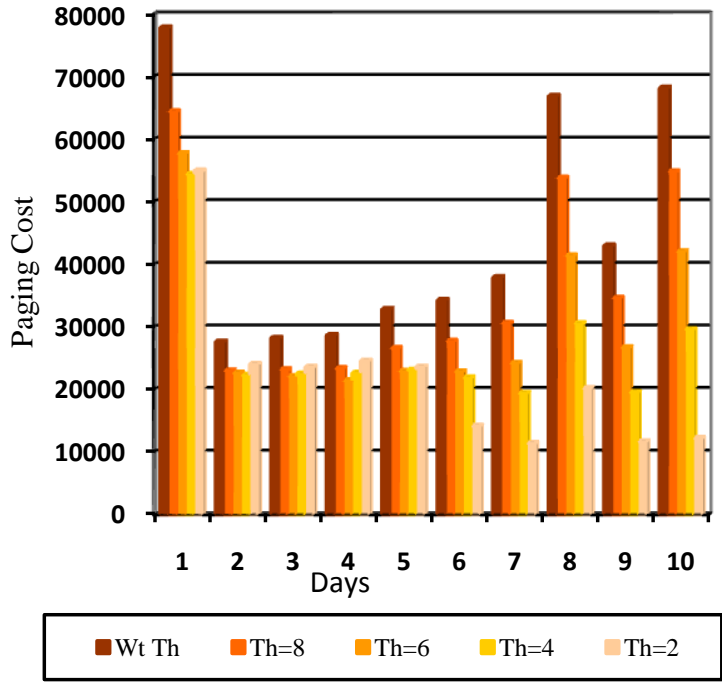

Fig 3: Comparison of paging cost for different Th value.

Table 3 show paging cost data during simulation of 10 days of 500 mobile units with 225 cells. Table 3 and Figure 3 show comparison of paging cost during simulation when threshold is $2,4,6,8$ and without threshold day wise. The paging cost is minimum for threshold=2 and is maximum for without threshold. Initially for 1 to 5 days there is little difference due to less prediction record but as days goes, difference becomes very high Above studying effect of varying threshold value on paging cost shows that rather than increasing, the paging cost decreases with decreasing threshold value because of left shift caching scheme to sort array. It shows that even when small threshold value is used, proposed scheme keeps page hit ratio substantially high by correct predictions and therefore whole LA is paged rarely thus the paging cost is minimized when smaller threshold is used.
Table 4. Paging Cost comparison with dynamic LM

\begin{tabular}{|c|c|c|c|}
\hline Days & Original & Dynamic Algorithm & Our Algorithm \\
\hline 1 & 110 & 80 & 54 \\
\hline 2 & 220 & 160 & 78 \\
\hline 3 & 380 & 230 & 101 \\
\hline 4 & 510 & 300 & 125 \\
\hline 5 & 640 & 380 & 149 \\
\hline 6 & 780 & 440 & 163 \\
\hline 7 & 920 & 520 & 174 \\
\hline 8 & 1020 & 600 & 194 \\
\hline 9 & 1180 & 660 & 205 \\
\hline 10 & 1300 & 740 & 217 \\
\hline
\end{tabular}

Table 4 shows paging cost values and Figure 4 shows paging cost comparison with dynamic location management scheme [3] and timer algorithm [23] with our algorithm. Results of dynamic location management scheme are achieved by simulation purpose we have assumed a cellular network of 100 cells where cells are square in shape. So below results are obtained by simulate our method with 100 cells from 225 cells and 50 mobile units.

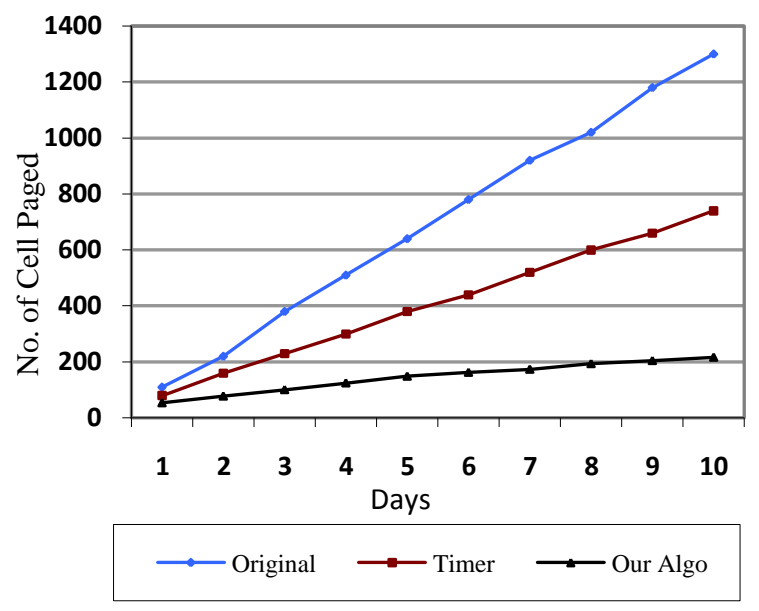

Fig 4: Comparison No. of cell page with dynamic LM

\subsection{Number of Page Hit against AMPuMM and CKbP}

As mention in above section proposed algorithm keeps hit ratio high. As well as fig. 5 compare result with existing method activity based mobility prediction using markov modeling (AMPuMM) [18] and CKbP method [22].

This comparison shows efficiency of predictions made by proposed scheme. When threshold value is $\mathrm{Th}=8$. Hit ratio obtained during simulation for different threshold value is given in Table 5. This comparison shows efficiency of predictions made by proposed scheme. If a MT found in predicated cell ID and there is no need to search that MT it called a hit if MT not found in predicated cell ID then normal paging is applied to search current location of MT it called miss. More page hit increases hit ratio. So efficiency of hit can be measure by hit ratio. A hit ratio is division of total call arrived by total no. of hit for particular day. 


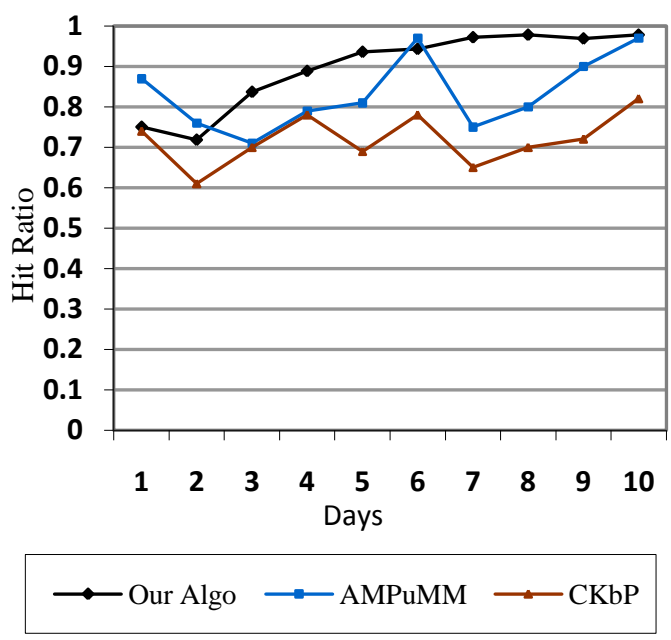

Fig 5: Performance improvement of proposed algorithm with AMPuMM and CKbP algorithm.

Table 5. Hit ratios for different Threshold values

\begin{tabular}{|c|c|c|c|c|c|}
\hline Days & Without Th & Th=8 & Th=6 & Th=4 & Th=2 \\
\hline 1 & 0.999 & 0.750 & 0.550 & 0.422 & 0.296 \\
\hline 2 & 0.999 & 0.719 & 0.306 & 0.216 & 0.261 \\
\hline 3 & 0.999 & 0.837 & 0.423 & 0.234 & 0.321 \\
\hline 4 & 1.000 & 0.888 & 0.537 & 0.248 & 0.794 \\
\hline 5 & 1.000 & 0.936 & 0.692 & 0.415 & 0.789 \\
\hline 6 & 1.000 & 0.943 & 0.795 & 0.540 & 0.820 \\
\hline 7 & 1.000 & 0.971 & 0.890 & 0.790 & 0.900 \\
\hline 8 & 1.000 & 0.978 & 0.951 & 0.896 & 0.954 \\
\hline 9 & 1.000 & 0.969 & 0.939 & 0.897 & 0.963 \\
\hline 10 & 1.000 & 0.978 & 0.957 & 0.940 & 0.991 \\
\hline
\end{tabular}

\subsection{Optimal Threshold decision}

The above results confirm the effectiveness of this method compare to existing AMPuMM and CKbP method for real time in mobile services. But more prediction value, searched for more paging, so it increases total average paging cost. Hence high threshold value leads to search MT unit in many cell IDs. It is again time wasting and cost increasing process. Therefore here is threshold value used for maintain page hit ratio. But if threshold value is to less hit ratio comes down rapidly for smaller threshold values. Thus threshold value helps to maintain hit to miss ratio. If page hit to miss ratio high then its total paging cost will also high. As shown in following fig. 6 . How page hit to miss ratio helps to maintain a good threshold value and it makes easy to find optimal threshold value which satisfied both total paging cost and hit ratio.

\subsection{Total Location Management Cost}

Location management cost is the summation of location update cost and paging cost, where the location update cost can be calculated by using following formula:

Location Update cost $=$ Unit Location Update cost $\times$ Number of Location Updates

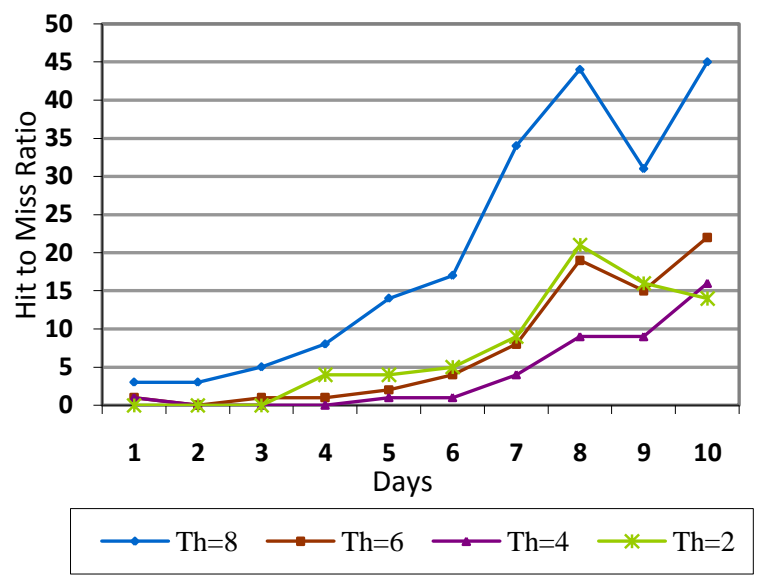

Fig 6: Page Hit to Miss Ratio for various threshold.

It is believed that the cost of a LU operation is ten times more than the cost of a paging operation [12]. In the light of this fact, value of unit location update cost has been taken as 10 . Location management cost can be determined using following formula $[11,13]$ :

LM cost $=$ Total Location Update cost + Total Paging cost

Fig. 7 shows comparison between location management costs when threshold $=2,4,6,8$ and without threshold. The location update cost is minimum for threshold=2 and is maximum for without threshold. It shows that even when small threshold value is used, proposed scheme keeps the location management cost considerably low. Results reveal that proposed scheme achieves better performance by minimizing location management cost. It shows effectiveness of paging cost, which also helps to decrease total cost. Where registration cost is fixed because of registration done only every mid night one time in a day so registration cost becomes almost constant. This results shows proposed scheme keeps the location management cost considerably low.

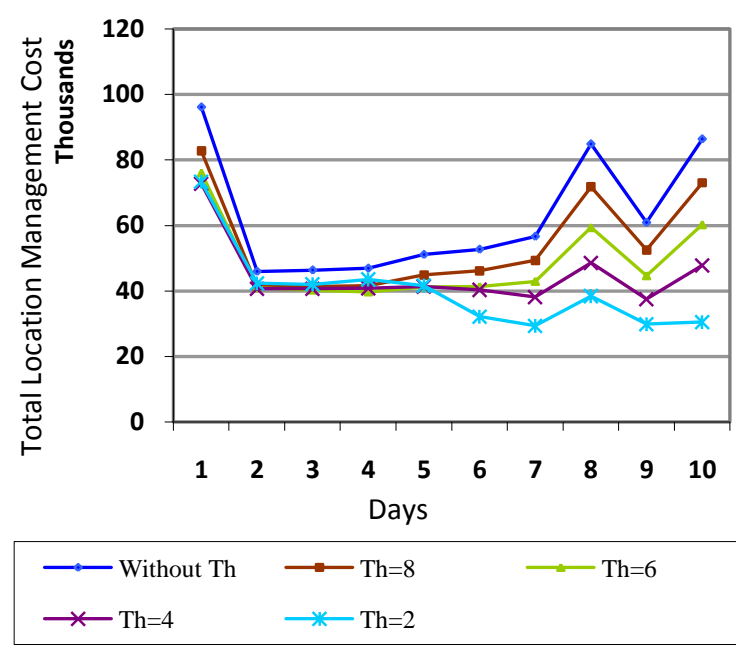

Fig 7: Total paging cost for various Threshold values.

Table 6 shows paging cost values and Figure 8 shows total LM cost comparison with dynamic location management scheme [3] and timer algorithm [23] with our algorithm. Results of dynamic location management scheme are achieved by simulation purpose we have assumed a cellular network of 100 cells where cells are square in shape. So 
below results are obtained by simulate our method with 100 cells from 225 cells and 50 mobile units.

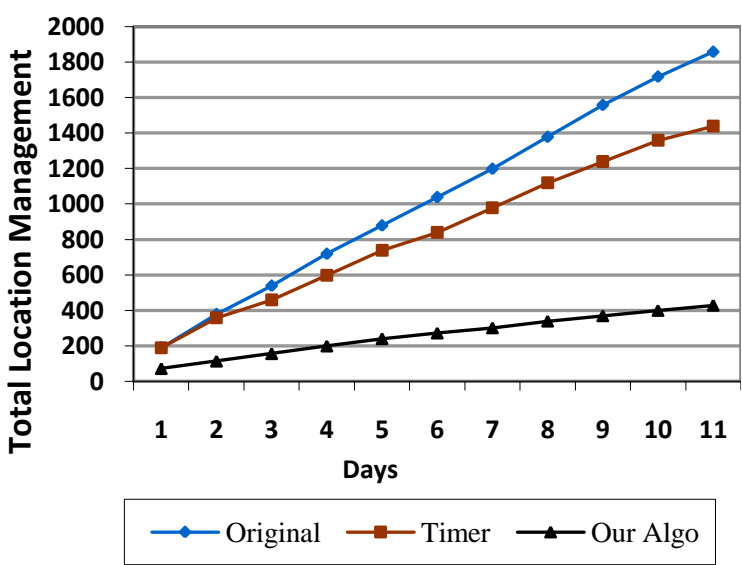

Fig 8: Comparison Total LM cost with Timer and Original LM

Table 6. Total LM cost comparison with dynamic LM

\begin{tabular}{|c|c|c|c|}
\hline Days & Original & $\begin{array}{c}\text { Dynamic } \\
\text { Algorithm }\end{array}$ & $\begin{array}{c}\text { Our } \\
\text { Algorithm }\end{array}$ \\
\hline 1 & 190 & 190 & 73 \\
\hline 2 & 380 & 360 & 115 \\
\hline 3 & 540 & 460 & 157 \\
\hline 4 & 720 & 600 & 200 \\
\hline 5 & 880 & 740 & 241 \\
\hline 6 & 1040 & 840 & 273 \\
\hline 7 & 1200 & 980 & 302 \\
\hline 8 & 1380 & 1120 & 340 \\
\hline 9 & 1560 & 1240 & 370 \\
\hline 10 & 1720 & 1360 & 400 \\
\hline
\end{tabular}

\section{CONCLUSION}

Mobile users move anywhere in mobile networks, so location registration for call tracking is always needed. The Timer Based Location Management in Cellular Network Using Cache Memory is free from maintaining large user history. This paper presented a new location management scheme using mobility information of users in cellular mobile networks, which adapts the prediction for locating mobile users according to their mobility patterns and call arrival frequency.

During simulation, user movements were kept neither fully random nor fully predefined and results prove that the proposed scheme predicts user location with high accuracy. The average hit ratio result comparison is AMPuMM algorithm gives 0.8445 and proposed algorithm maintains average hit ratio at 0.9043 . It shows that proposed algorithm is $6 \%$ more efficient with existing methods.

Table 7 show first ten days average values for various cost generated with different threshold values during simulation. This average will be better optimizing for upcoming days. This table shows simple relations between paging cost, total location management cost and hit ratio. This relation is very helpful for deciding optimum threshold value by observing total LM cost according to Threshold value to get better hit ratio.

Table 7. Overall average data for different threshold

\begin{tabular}{|c|c|c|c|c|c|}
\hline Average & $\begin{array}{c}\text { Without } \\
\text { Th }\end{array}$ & $\mathbf{T h = 8}$ & $\mathbf{T h}=\mathbf{6}$ & $\mathbf{T h = 4}$ & $\mathbf{T h = 2}$ \\
\hline $\begin{array}{c}\text { Paging } \\
\text { Cost }\end{array}$ & 44530 & 36225 & 30399 & 26605 & 22052 \\
\hline $\begin{array}{c}\text { Total } \\
\text { LM } \\
\text { Cost }\end{array}$ & 62786 & 54481 & 48655 & 44861 & 40288 \\
\hline $\begin{array}{c}\text { Hit } \\
\text { Ratio }\end{array}$ & 0.9997 & 0.9043 & 0.70384 & 0.55979 & 0.70898 \\
\hline
\end{tabular}

Using the longitude and latitude information extracted from the GSM traces, the present cell id could be found. This GSM trace information could be used for next generation of mobile like $3 \mathrm{G}$ and 4G.But extra cache memory is needed in mobile unit which increases the cost of mobile terminal.

\section{REFERENCES}

[1] J. Gil, Y. Chan, C. Hwang, D. Park, J. Shon, and Y. Jeong, "Restoration Scheme of Mobility Databases by Mobility Learning and Prediction in PCS Networks," IEEE J. Selected Areas in Comm., vol. 19, no. 10, 2001, pp. 1962-1973.

[2] E. Cayirci and I.F. Akyildiz, "User Mobility Pattern Scheme for Location Update and Paging in Wireless Systems," IEEE Trans. Mobile Computing, vol. 1, no. 3, July-Sept. 2002, pp. 236-247.

[3] Vimal Bhatt and Digvijay Singh Khatri,. "Timer Based Dynamic Location Management”, IEEE, 2004

[4] Ahadur Rahaman, Jemal Abawajy and Michael Hobbs, "Taxonomy and survey of location management systems", 6th IEEE International Conference on Computer and Information Science, 2007.

[5] Bhavneet Sidhu and Hardeep Singh, "Location management in cellular networks", WASET, 2007, pp. 314-319.

[6] Babak D. Beheshti, "Review of location management in cellular networks", IEEE, 2007.

[7] Kenneth W.Shum and Chi Wan Sung, "Kalman-filter based predictive location management for PCS networks", IEEE, 2003, pp. 03-15.

[8] Ian F. Akyildiz and Joseph S. M. Ho, "On location management for personal communications networks", IEEE Communications Magazine, Sept. 1996.

[9] Sherif Akoush and Ahmed Sameh, "The Use of Bayesian Learning of Neural Networks for Mobile User Position Prediction," 7th International Conference on Intelligent Systems Design and Applications, 2007, pp. 441-446.

[10] Ashish Goel, Navankur Gupta, Prakhar Kumar, "A speed based adaptive algorithm for reducing paging cost in cellular networks", IEEE, 2009, pp. 22-25.

[11] Tae Sung Kim, Kang Jin Yoon, and Young Yong Kim, "A Predictive Location Based Relaying scheme for hybrid Cellular and Ad-hoc Systems," International Conference on Multimedia and Ubiquitous Engineering, 2007. 
[12] Pratap S Prasad and Prathima Agrawal, "Mobility Prediction for Wireless Network Resource Management," 41st Southeastern Symposium on System Theory, 2009, pp. 98-102.

[13] Shafagh Alikhani, Marc St-Hilaire and Thomas Kunz, "iCCA-MAP: A New Mobile Node Localization Algorithm," IEEE International Conference on Wireless and Mobile Computing, Networking and Communications, 2009.

[14] T.X. Brown, S. Mohan, "Mobility management for personal communications systems," IEEE Trans. Vehicular Technology, vol. 46, no. 2, pp. 269-278, May 1997.

[15] Y. Fang, Chlamtac and Y. Lin, "Portable movement modeling for PCS Networks," IEEE Transactions on Vehicular Technology, vol.49, no.4, pp.1356-1363, July 2000 .

[16] Y. Fang, Chlamtac and H. Fei, "Analytical results for optimal choice of location update interval for mobility database failure restoration in PCS networks," IEEE Transactions on Parallel

[17] M.S. Sricharan, V. Vaidehi, P.P. Arun, "An Activity based Mobility Prediction Strategy for Next Generation wireless Networks", IEEE, IFIP, Wireless Optical and communication Networks, 2006.

[18] R.V. Mathivaruni and V. Vaidehi "An Activity Based Mobility Prediction Strategy Using Markov Modeling for Wireless Networks" WCECS 2008.

[19] Martin, E., "A Graphical Study of the Timer Based Method for Location Management with the Blocking Probability" (WiCOM), 2011.

[20] Chien-Sheng Chen; Szu-Lin Su; Chyuan-Der Lu, "Geometrical positioning approached for mobile location estimation", IEEE 2010 , Page(s): 268 - 272.

[21] Munadi, R.; Ismail, M.; Abdullah, M.; Misran, N.;" Location management cost reduction using fuzzy logic in cellular radio network",IEEE 2011, Page(s): 165 - 169

[22] Nancy Samaan and Ahmed Kormouch, "A Mobility Prediction Architecture Based on Conceptual Knowledge and Spatial Conceptual Maps", IEEE Transactions on Mobile Computing, vol 4, no 6, pp. 537-551, 2005.

[23] Ajit Pal and Digvijay Singh Khati, "Dynamic Location Management with Variable sue Location Areas",2001 International Conference on Computer Networks and Mobile Computing . 